\title{
H1N1: Revealing the Collective Resolve of Medicine and Public Health
}

\author{
Italo Subbarao, DO, MBA, Lewis Rubinson, MD, PhD, and James J. James, MD, DrPH, MHA
}

$\mathrm{I}$ $n$ just 6 months since the isolation of 2009 H1N1 influenza, the world is facing the first influenza pandemic in more than 3 decades, and there remains almost no refuge from human illness. To date, the illness is at most a nuisance for the majority of people, whereas for a small percentage of children, pregnant and peripartum women, and other young adults across the globe, severe critical illness and death have been reported. These diametrically opposed clinical experiences have made it particularly difficult to mount and sustain the appropriate level of public health, medical, and community member activity to minimize influenza impact for all. Nevertheless, lessons from local pockets of intense disease activity in addition to the critical illness experienced in temperate-climate southern hemisphere countries have compelled many public health and clinical experts to recognize that $2009 \mathrm{H} 1 \mathrm{~N} 1$ is not just seasonal influenza. Combating this illness, however, is no small feat: Vaccine needs to be developed and distributed in parallel with seasonal influenza vaccine, infection control guidance in health care settings needs to be agreed upon, and sufficient antivirals need to be available and include novel agents as contingencies for limited effectiveness, resistance, and poorly tolerated routes of administration. In addition, health care systems must develop novel mechanisms to meet a possible increase in clinical demand but do so within the restraints of economic market forces that tend to limit surge capacity. Most important, proper strategies and communication to engage our fellow community members to work toward a common good need to be developed and implemented.

Thus far, this pandemic has revealed the extraordinary character and resilience of the medical and public health communities as they have risen to meet and overcome this challenge. Since the first wave of the pandemic, all of the components of the medical and public health care systems have dutifully and diligently worked together to gain valuable insight into the characteristics and peculiarities of this novel strain of influenza virus. Clinicians, epidemiologists, and virologists around the world have worked tirelessly to manage and investigate this new disease. Many of their early efforts led to medical publications providing preliminary insights regarding the suscep- tibility of children and pregnant women, as well as the potential challenges regarding critical care patients. As the first wave crested, the US federal government, academia, professional societies, and the private sector attempted to address salient and challenging issues in preparing for the second wave. Forums addressing the appropriate respiratory mask protection for health care workers, crisis standards of care criteria, and vaccine prioritization were convened and have provided frontline caregivers timely guidance for the succeeding waves. The integrated efforts of the National Institutes of Health, the Biomedical Advanced Research Development Authority, the Food and Drug Administration, along with the pharmaceutical industry and academia in working around the clock to develop, test, and distribute an $\mathrm{nH} 1 \mathrm{~N} 1$ vaccine in time for the second wave is, to date, the major achievement in this endeavor.

These achievements could not have been attained without earlier sustained investments in pandemic influenza preparedness. Notably, many changes in the evolution of our public health and medical care delivery systems since the last influenza pandemics inadvertently left considerable vulnerabilities for responding to even a moderately severe pandemic. Fortunately, the significant preparedness efforts of the past decade have reversed many of these weaknesses. One overarching and contributory factor in this success lay in the foresight of federal and private sector preparedness grant programs that stimulated and encouraged regional collaboration between the private and public sectors, developing the essential infrastructure and relationships required for this challenging time. These activities were also mirrored by similar organizations around the world. This renewed and integrated response infrastructure coupled with the tremendous surge in a broad range of public health and medical activities since 2001 have significantly increased the resilience of many countries against whatever this pandemic may bring.

One extremely important factor that has become apparent from the current experience is the need for consistent and effective risk communication; otherwise, regardless of the effectiveness of countermeasures, public health interventions are significantly mitigated in the absence of public understanding and acceptance. As such, pandemic H1N1 has become a 
victim of the 24/7 hypermedia news cycle. Initially, of course, the disease was called "swine flu," but then came "Mexican flu," "swine origin novel influenza," "2009 pandemic H1N1," and so forth, until finally, to the public, it became as it began, swine flu. We cannot afford to let semantic issues distract us from the relevant communication related to public health interventions. The editors of this issue strongly recommend an analysis and solution to the taxonomy shortfalls in hopes that risk communication would be significantly improved to enhance resilience for future pandemics.

It is in this spirit that we present our special issue on H1N1, which is intended to improve our informed medical and public health response. Herein, frontline responders will find scholarly articles and commentaries regarding standards of care considerations for both adult and pediatric patients (Cadigan et al and Kanter and Cooper), respiratory mask protection for health care workers (Radonovich et al), strategies for point of distribution of medical countermeasures (Hupert et al), and a historical commentary provided by John
Barry. More important, it is another tool to better inform us going forward.

Although many challenges and uncertainties will persist in dealing with this novel strain, previous calamities such as smallpox and polio have taught us a similar lesson: An integrated and resilient medical and public health community is the essential foundation to a successful response and to the protection of the public health.

\section{About the Authors}

Drs Subbarao and Rubinson are coeditors of this issue; Dr James is the editor-inchief of Disaster Medicine and Public Health Preparedness.

Received and accepted for publication October 21, 2009.

\section{Authors' Disclosures}

The authors report no conflicts of interest.

ISSN: 1935-7893 (C) 2009 by the American Medical Association and Lippincott Williams \& Wilkins.

DOI: 10.1097/DMP.0b013e3181c74bba 